\title{
Design and Development of SHAD - A Small Hovering AUV with Differential Actuation
}

\author{
Carlos S. Gonçalves, Bruno M. Ferreira, Aníbal C. Matos \\ INESC TEC, Faculty of Engineering, University of Porto \\ Rua Dr. Roberto Frias, 378 \\ 4200-465 Porto, Portugal \\ cgoncalves@fe.up.pt, bm.ferreira@fe.up.pt, anibal@fe.up.pt
}

\begin{abstract}
This paper presents the design and development of a new Autonomous Underwater Vehicle (AUV). SHAD, which stands for Small Hovering AUV with Differential actuation, is a torpedo shaped vehicle that was conceptually designed to navigate in challenging volumes. It brings to the scene of submarine robotics a different model and new design of AUV. The small size, the light weight and the high maneuverability of this AUV were among the most important features that can make the SHAD an option to applications where other models have difficulties. This paper details the design and the development of SHAD and presents experimental results from sensors and actuators testing as well as vehicle navigation.
\end{abstract}

\section{INTRODUCTION}

From several applications in the domain of underwater robotics, emerged the need to design an Autonomous Underwater Vehicle (AUV) with specific characteristics enabling navigation in constrained volumes of water. Some examples include rivers, lakes, offshore structures or test tanks with small dimensions. To address those challenges, we have developed a new, small-sized AUV with hovering capabilities that can be easily reconfigured: Small Hovering AUV with Differential actuation (SHAD).

The design and implementation of the SHAD AUV resulted from the integration of several modules and components previously developed, based on the experience of INESC TEC in robots design and development.

The AUV was designed so that it could operate both in indoor and outdoor environments. Further to the capability of navigating in constrained volumes of water, logistics concerns were kept in mind when developing SHAD's design. Its dimensions and weight are advantageous, as it is man portable and easily transportable. One operator, alone, can carry it to a car or to a small boat and deploy it easily. All the inverse logistic processes are similarly easy and require the same human and material supporting means.

Smaller vehicles have been developed. The LAUV [1] is a good example of those small-sized AUV that are one-man portable and allow smaller logistics and simpler handling, thanks to their dimensions $(0.15 \mathrm{~m}$ hull diameter; $1.2 \mathrm{~m}$ standard length) and light weight $(16 \mathrm{~kg})$. However, its motion capabilities, along with that of similar small-sized vehicles, restrict its navigation in confined volumes as the attitude and
TABLE I

PROJECT REQUIREMENTS

\begin{tabular}{|l|l|}
\hline Length & $\leq 1 \mathrm{~m}$ \\
\hline Diameter & $\leq 0.1 \mathrm{~m}$ \\
\hline Weight & $\leq 10 \mathrm{~kg}$ \\
\hline Transportation & Must fit in a car trunk \\
\hline Launch and recovery & Small boat, shore \\
\hline Handling & One-man portable \\
\hline Maneuverability & 4 DOF \\
\hline Depth rating & $\geq 10 \mathrm{~m}$ \\
\hline Reconfiguration capability & Modular, Expandable \\
\hline
\end{tabular}

the depth cannot be controlled independently of the forward motion.

Other examples of AUV have more than $1.5 \mathrm{~m}$ of length and $30 \mathrm{~kg}$ in weight [2]-[4]. Though they are of a smaller size, for certain situations these AUV prove to be large and heavy in logistic concerns. Also, they have high difficulty in maneuvering in small or structured places. It is here that the SHAD offers a complimentary and competitive edge thanks to its characteristics. The combined geometry, weight and actuation capabilities provide high maneuverability to SHAD, which is of special importance in small volumes.

\section{REQUIREMENTS}

The operation of most AUVs often implies complicated logistics to deploy and recover them. Furthermore, their operations in small volumes may be impossible due to their dimensions and/or to their motion capabilities, namely for what concerns degrees of freedom. With this in mind, the requirements for the design of SHAD have been established and are presented in Table I.

Additionally, the vehicle geometry and method of actuation must endorse the portability of models and developed software.

\section{CONCEPT AND DESIGN}

Conceptually, SHAD is subdivided into three major blocks: the waterproof part, the wet exterior part and the mediation part of both. With the exception of some sensors dedicated to 
marine robots (for example, pressure sensor) and waterproofing components, the subsystems and components that form these blocks are the common for the design and development of general robots.

At the centre of the AUV, a hollow and watertight compartment will be used to house all electrical components and batteries that are used to drive the vehicle. This compartment is sealed off using two sealing caps that are attached to the ends of the central cylinder. They are designed in such way that the electrical components and batteries can be accessed with ease, simply by removing one of these sealing caps and withdrawing the board on which all these components are placed. Though, the AUV will incorporate a waterproof plug with easy access for loading batteries.

These covers will also be the medium through which all cabling will be passed in a sealed, watertight manner to the inside of the cylinder. The remaining structure expands from this core through various mechanical parts that govern the strength of the whole structure. The design is of a modular nature where the structure can be expanded upon using parts that are developed in isolation and connected to the structure as required. This approach is followed for all constituents making up the AUV or payload.

SHAD is modeled on a torpedo-like geometry that will offer its design a streamlined flow of water across the body during submerged operation. This vehicle will have the ability to dive vertically down a column of water and move itself along a constant depth. The vehicle will also have four thrusters for propulsion, two of them induce "horizontal" forces in the longitudinal direction and the other two induce "vertical" force to move in the heave direction. The idea of a hovering AUV and the thrusters configuration was partially inspired in the MARES AUV [2], also developed by INESC TEC. Other examples of hovering AUVs include [3] and [4]. The differential actuation is obtained by making the propellers of each pair of motors, horizontals and verticals, actuate with different forces to obtain torques about the center of mass. Such a differential actuation enables controlling the pitch with the vertical thrusters and the yaw with the horizontal ones. With this thruster configuration, SHAD has four degrees of freedom: surge, heave, pitch and yaw.

The reduced size and the streamlined body of SHAD minimize the viscous damping, thus making it efficient. In addition, at extreme ends of the body, the nose and tail are designed to have a particular shape for that.

The depth to which the AUV descends is measured by a pressure sensor.

Its orientation will be obtained through inertial sensors and its position estimate using the fusion of information gathered on the vehicles inertia, its performance and a motion model used in the vehicles programming.

On the surface, the position of the AUV can be detected and corrected through the use of GPS technology. Serial communication is used between subcomponents of the AUV inside the central cylinder. Above the surface of the water, information is transmitted wirelessly.

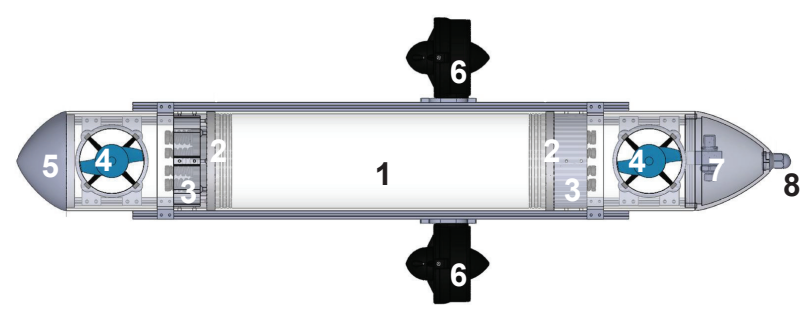

Fig. 1. SHAD top view

\section{DEVELOPMENT}

The schematical drawing of the different components of the AUV are shown in Figure 1, with the following correspondence:

1. Pressure hull, 2. End-caps, 3. Structural supports , 4. Vertical thruster modules, 5. Nose, 6. Horizontal thrusters, 7. Pressure sensor, 8. Batteries charging terminal.

\section{A. Mechanical design}

The components of the general structure of the AUV (in component form) will now be described. After all components are mounted and interconnected, two aluminium frames of a square profile $(1 \times 1 \times 60 \mathrm{~cm})$ are placed laterally along the body of the vehicle. These frames are fixed using special joints that are designed to ensure that the frame of the vehicle (pressure hull, end-caps and structural supports) is of a robust enough nature to mitigate and restrict movement in the longitudinal plane. This is to ensure that the central cylinder does not open.

Though these frames and joints offer some restriction to rotational movement, the friction between the contacts of the o-rings (found in the end-caps) and the pressure hull account for the majority of this restriction.

The above mentioned frames are also designed in such way that the position of horizontal motors of the vehicle can be varied along its length.

1) Pressure hull: this central cylindrical compartment serves as a sealed container in which components that cannot come into contact with water are housed. It is manufactured using transparent acrylic perspex and stores all electronic components and batteries. These components are placed on a structure made of aluminum frames, plastic and acrylic. Additionally, it is attached to the back lid (end-cap) of the cylinder, enabling the withdrawal of the platform for easy access to these electrical components by sliding the entire structure along the interior of the cylinder.

2) End-caps: These parts are made of polyacetal plastic (POM) and are the lids attached onto either ends of the pressure hull to ensure a watertight compartment inside. This is accomplished using o-rings. The end-caps serve to demarcate the dry and wet areas of the vehicle. In order to connect the necessary cables to the electrical components housed inside the cylinder, without the unwanted infiltration of water, glands are fixed to the end-caps to ensure that this cabling is connected in a watertight manner. 
3) Structural supports: The structural supports of the vehicle, under normal operation, will undergo rotational motion. These are made of POM plastic, crafted in a linear finish, and are fastened using a screw on the exterior face of either end-cap. Their primary function is to enable the rest of the exterior modules and components of the AUV to be fixed to the central cylinder. After, there are square aluminium tubes that support themselves on the internal lateral indentations of the structural support which will become the base on which all other modules of the AUV will be mounted.

4) Vertical thrusters modules: The vehicle has two modules that cater for vertical thrust. To assemble these modules, a motor and the propeller were harvested from a commercial solution and attached to a duct and an acrylic shell designed and developed along the project. They are an example of modules that can be mounted in the structural supports.

5) Nose design: These parts have a geometry based on the Von Karman Ogive curve with the intention of mitigating as much as possible the viscous friction that the vehicle will encounter during motion, thereby improving its efficiency. The tail of the vehicle is larger than the nose for hydrodynamic efficiency proposes (form of a tear drop shape) and due to the necessity of housing in it the pressure sensor and the battery charging plug. Some components were created using a 3D printer and coupled to a commercially available waterproof connector, afforded the project of a quick-fit solution for the charging terminal of the batteries. These are located at the extreme end of the SHAD tail, where there is an aperture to fit the terminal.

\section{B. Actuators and Sensors}

1) Thrusters: SHAD is propelled by four commercially available underwater thrusters designed specifically for marine applications. Each one is composed of a brushless electric motor of $130 \mathrm{~W}$ maximum power and provides a thrust of $23 \mathrm{~N}$ maximum forward force. Two of these motors, those used for horizontal motion, are used as sold originally whereas the other two have had the motors and propellers stripped and assembled into a custom designed structure to fit the streamline shape of the vehicle.

2) Pressure Sensor: In order to determine the depth at which the SHAD will be, a pressure sensor fit for industrial applications was modified to have a watertight electrical cable and connection to the sensor. The sensor supports pressures of up to 6 bar; features an accuracy of $0.25 \%$ and has a typical analogue output of 4 to $20 \mathrm{~mA}$.

\section{Batteries}

The electrical energy required for the operation of SHAD is sourced from two Lithium-Polymer (Li-Po) batteries of $11.1 \mathrm{~V}$ connected in parallel. Each battery has a $3.7 \mathrm{~A} \mathrm{~h}$ capacity and a maximum continuous discharge current of $90 \mathrm{~A}$.

\section{Electronics}

1) Battery controller: A printed circuit board (PCB) was developed and integrated to extract information regarding the

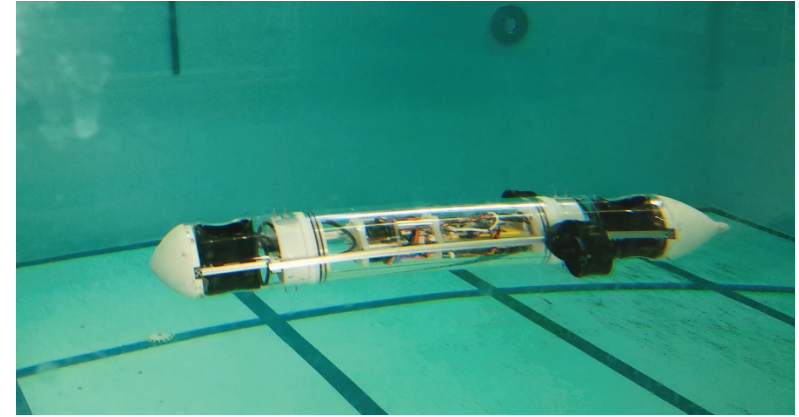

Fig. 2. SHAD in test tank

batteries. For example, the total supply voltage in each cell as well as the total current being drawn from the batteries. This information can then either get processed in a microprocessor also present on this PCB or downloaded to the central computer via a serial port. The power supply is cut should the voltage of a cell drop below a predefined value.

2) Central embedded computer: Raspberry Pi 2 Model B. This ARM-based single board computer has a quad core processor running Linux Debian. Further to its functionalities, this board was chosen due to its number of device interfaces, namely USB ports, a serial port and several digital inputs/outputs.

3) Electronic drive module: This module constitutes various mechanical parts that support 4 independent motor drives that feed and control the motors of the vehicle using Electronic Speed Control (ESC). Additionally, the electronic drive module also contains custom designed PCB that acts as a central gateway for command between the computer and the ESC module.

4) DC/DC converters: These are used to feed the low power electronics of a different rated supply voltage to that of the batteries.

5) Attitude heading reference system (AHRS): The attitude sensor is composed of the following microelectromechanical systems (MEMS): accelerometer, gyroscope and magnetometer. The AHRS already contains a microcontroller running an Extended Kalman Filter (EKF) that combines all measurements and readings from the sensor. In just one second, the microcontroller is capable of estimating the orientation on the vehicle 500 times. This corresponds to the the attitude (roll and pitch angles relative to the gravity vector) and heading (angle with respect to the geographic North - azimuth).

6) Pressure sensor acquisition circuit: This circuit is made up of hardware developed that reads the analogue output current of the pressure sensor and conditions this signal before converting it to a digital signal to be sent over serial communications to the central computer. In order to achieve the intended operation of this hardware, the necessary software was also developed. This includes a driver for the device (located on the board) as well as a driver on the central computer that enables interaction with all other layers of software. 


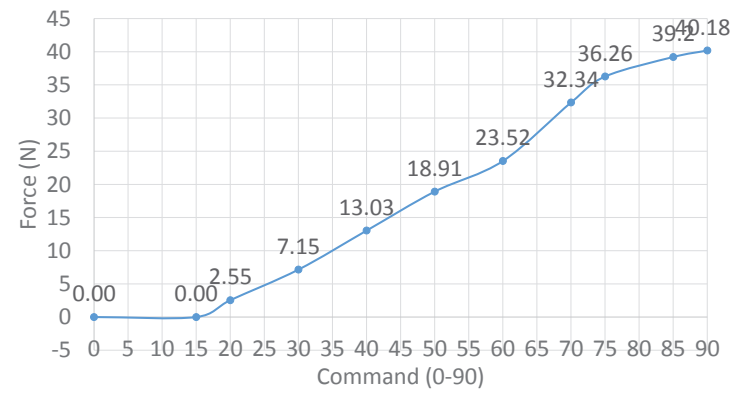

Fig. 3. Force vs. Command for a pair of thrusters

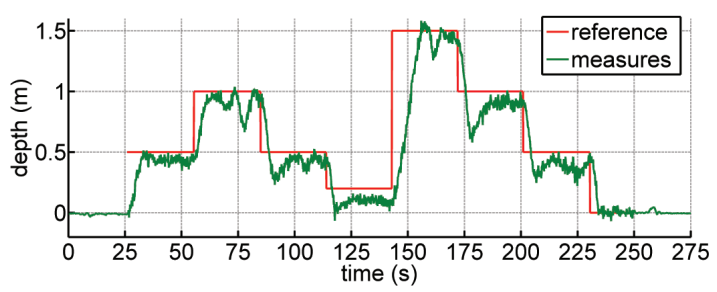

(a) Results of hovering mission

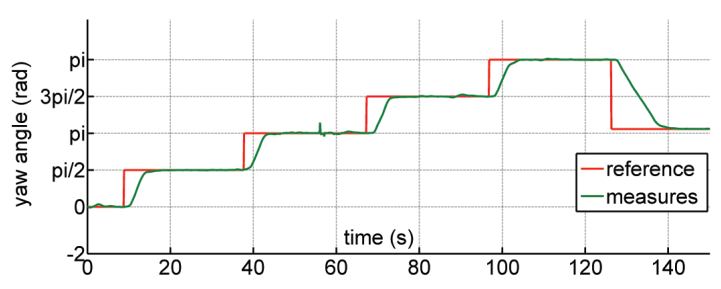

(b) Results of yaw angle test

Fig. 4. Results of SHAD tests

\section{Characterization OF THRUSTERS}

For control purposes, it is of utmost importance to characterize the actuation. In fact, as it is impossible to measure the forces applied by the thrusters, it is customary to use an openloop model of the force. Therefore, the relationship between the force and the command has to be established. Such relation is presented in Figure 3 along with an analysis of the power consumption. A special setup, involving the SHAD fixation to a scale, was mounted to measure the force exerted by the motors.

By means of this experiment, the relationship between the commands and the actual force performed by the thruster was extracted. One can see that this relation is approximately linear in the region from command 15 to 60 .

The characterization was done to one pair of motors, with forward and reverse rotation, and assumed to the other pair, since all of them are the same model. From the obtained data, it is clear that forward movement is more efficient than the reverse one, as expected.

\section{TESTS AND RESUltS}

Several tests were performed in order to validate the entire solution and to assess the performances of the AUV.
Experiments were planned and executed in a test tank in our facilities (Figure 2) by missions programmed into the vehicle. The results of the SHAD global test missions present measurements in terms of depth for the hovering mission and yaw angle measurements for the attitude mission. The hovering test mission consisted of a dive at a variable depth reference. The mission was programmed so that the depth reference varies in steps, as depicted in Figure 4a. Each step is programmed to last 30 seconds. The measured results show that the vehicle achieved a dive maximum of $1.5 \mathrm{~m}$ crossing through $0.5 \mathrm{~m}$ and $0.2 \mathrm{~m}$ alternately before it rises to the surface in steps of $0.5 \mathrm{~m}$.

The results show that SHAD was able to track the reference with less than $10 \mathrm{~cm}$ of error in steady state, with the exception of larger peaks that were the consequence of a dead zone at low rotation speeds, that made one of the vertical thrusters stop during short intervals.

The attitude test mission was conducted at a constant depth ( $1 \mathrm{~m})$ and variations in yaw angles according to the mission references as shown in Figure 4b. Each heading reference step was programmed to last 30 seconds. The motion of the SHAD can be described by semi circles, where it moves in steps of $90^{\circ}$ and finally it rotates $180^{\circ}$ in the opposite direction.

The results demonstrate the SHADs capability to accurately track the yaw reference with an error typically under $1^{\circ}$ in steady state.

This experimental results demonstrate also the stability of SHAD.

\section{CONCLUSION}

The work presented in this paper conducted to the design and the implementation of a new AUV. In general terms, results in an AUV with $0.12 \mathrm{~m}$ of diameter (conditioned by the acrylic perplex standard size) and $0.90 \mathrm{~m}$ of length combined with $6.0 \mathrm{~kg}$ of weight. The vehicle's depth rating is currently estimated to be $20 \mathrm{~m}$, but can be easily increased. The small, lightweight AUV SHAD was conceptually thought to navigate in confined volumes but can also be deployed in open, outdoor environments. The hovering capability offers unique advantages in those environments. After the vehicle was completely implemented, validation and performance tests were performed. These results have demonstrated that the vehicle is capable of precise positioning in the vertical axis, with a tracking error of about $10 \mathrm{~cm}$, that is effectively less than the vehicle diameter, and in the angular axes with an error approximately of $1^{\circ}$.

\section{REFERENCES}

[1] L. Madureira, A. Sousa, J. Braga, P. Calado, P. Dias, R. Martins, J. Pinto, and J. Sousa, "The light autonomous underwater vehicle: Evolutions and networking," in OCEANS - Bergen, 2013 MTS/IEEE, June 2013, pp. 1-6.

[2] N. A. Cruz and A. C. Matos, "The MARES AUV, a Modular Autonomous Robot for Environment Sampling," in OCEANS 2008, 2008, pp. 1-6.

[3] M. Radojevic, M. M. Nawaf, F. Maurelli, T. Tomassson, C. Wang, J. Clay, and Y. Petillot, "Nessie VI autonomous underwater vehicle," 2011.

[4] M. Carreras, C. Candela, D. Ribas, A. Mallios, L. Magí, E. Vidal, N. Palomeras, and P. Ridao, "Sparus II, design of a lightweight hovering $A U V$," in Martech 2013 5th International Workshop on Marine Technology. SARTI, 2013. 